\title{
CONTRA EL NOMINALISMO. HUMANISMO Y RENOVACIÓN DE LA FILOSOFÍA Y TEOLOGÍA EN PEDRO MARTÍNEZ DE OSMA (1424-80)
}

\author{
José Luis Fuertes Herreros \\ Universidad de Salamanca
}

\section{RESUMEN}

En torno al Concilio de Basilea (1431-49), y frente a la renovación nominalista, en la Universidad de Salamanca se promueve todo un movimiento de renovación humanista desde el campo de la filosofía y teología, teniendo en Pedro Martínez de Osma (1424-80) a uno de sus representantes más significativos. En este trabajo se muestra el significado de su obra, con influencias de L. Bruni y L. Valla, y se rescatan dos de sus textos más significativos desde esa lucha que enfrentaba al humanismo con el nominalismo.

Palabras clave: Humanismo, nominalismo, Renacimiento, filosofía escolástica, Pedro Martínez de Osma, Aristóteles, L. Bruni, L. Valla, Universidad de Salamanca.

\section{ABSTRACT}

Around the Council of Basel (1431-49), and opposing the nominalism renewal, in the University of Salamanca a whole new movement of humanistic renewal is promoted from the field of philosophy and theology, having in Pedro Martínez de Osma (1428-80) one of its most significant representatives. In this paper the meaning of his work, with influences from L. Bruni and L. Valla, is shown and two of his most significant texts are rescued from that fight which confronted the humanism against the nominalism.

Key words: Humanism, nominalism, Renaissance, scholastic philosophy, Pedro de Osma, Aristotle, L. Bruni, L. Valla, University of Salamanca.

\section{UNA APROXIMACIÓN A PEDRO MARTÍNEZ DE OSMA}

El interés por Pedro Martínez de Osma, o Pedro de Osma (1424-1480), ha ido en aumento desde que se celebrara el quinto centenario de su muerte en $1980^{1}$, aunque anteriormente Menández Pelayo ${ }^{2}$, Federico Stegmüller ${ }^{3}$, T. y J. Carreras y Artau ya habían reparado en él ${ }^{4}$ y

1 Pérez Rioja, J. A., Pedro Martinez de Osma (+1480). Homenaje en el V centenario de su muerte, Centro de Estudios Sorianos, CSIC, Soria 1980, que era publicación aparte de la revista Celtiberia y que había dedicado a Pedro Martínez de Osma, 30 (1980) 1-160.

2 Menéndez Pelayo, M., Historia de los heteredoxos españoles, $2^{\circ}$ edición, Madrid 1918 ,

3 Stegmüller, F., «Pedro de Osma. Ein Beitrag zur spanischen Universitäts-Konzils-und Kertzergeschichte», Römische Quartalscrift, 43 (1935) 205-66.

4 Carreras y Artau, T. y J.; Historia de la filosofia española. Filosofia cristiana de los siglos XIII-XV, Tomo II, Madrid 1943, 564-571. 
luego lo haría con nuevo impulso el profesor Klaus Reinhardt ${ }^{5}$. Posteriormente, los estudios se han ido sucediendo. Los propios de Klaus Reinhardt, junto con Horacio Santiago-Otero y $\mathrm{M}^{\mathrm{a}}$ Teresa Cardo ${ }^{6}$, los de José Labajos Alonso, publicando dos de sus más importantes obras ${ }^{7}$ y, últimamente, de Ana Cebeira ${ }^{8}$, nos han posibilitado advertir la importancia de este autor. Desde otras aproximaciones, también, la figura de Pedro de Osma, ha estado presente, entre las cuales cabe destacar, las de Vicente Muñoz $z^{9}$ Gabriel González ${ }^{10}$, y se muestra en la Historia de la Universidad de Salamanca, que bajo la dirección de Luis Enrique Rodríguez-San Pedro actualmente se está publicando ${ }^{11}$, así como en la nueva mirada que se está proyectando sobre las Escuela de Salamanca o sobre los estudios de la filosofía del Renacimiento en general ${ }^{12}$.

El haberme acercado, también, a Pedro Martínez de Osma desde diversos estudios sobre la filosofia del Renacimiento es lo que me ha movido a ofrecer traducidos, ahora en primicia, dos pequeños grandes textos de Pedro de Osma, Responsio ad quedam deliramenta duorum huius temporis verbosistarum y Dialogus'in quo ostenditur fundamenta humanae philosophie, quibus fulciuntur verbosiste, plerumque in theologia deficere, que Reinhardt y Santiago-Otero supieron rescatarlos y publicarlos en latín ${ }^{13}$, y a ellos acuden, también, José Labajos ${ }^{14}$ y Ana Cebeira $^{15}$. Sin duda que con esta traducción se habrá contribuido a una mejor comprensión de la filosofia del Renacimiento.

5 Reinhardt, K., Pedro de Osma y su comientario al símbolo "Quicumque», Estudios y Ensayos. Joyas bibliográficas, Madrid 1977.

6 Cardo, M ${ }^{\mathrm{a}} \mathrm{T}$, Santiago-Otero, H., Reinhardt, K., «Crítica y aportación de Pedro de Osma al método teológico», Humanismo, Reforma y Teología, Instituto Francisco Suárez, CSIC, Cuaderno 57, Serie Estudios 19, 1984. Santiago-Otero, H. y Reinhardt, K., Pedro Martinez de Osma y el método teológico: edición de varios escritos inéditos, CSIC, Madrid 1987.

7 Pedro de Osma y su comentario a la Metafisica de Aristóteles, edición crítica latina de José Labajos Alonso, Publicaciones de la Universidad Pontificia de Salamanca 1992, 479 pp.; y, Pedro de Osma. Comentario a la Ética de Aristóteles, edición crítica latina de José Labajos Alonso, Publicaciones de la Universidad Pontificia de Salamanca 1996, 657 pp. La primera de esta obras será citada por $C M$, y la segunda por $C E$.

8 Pedro Martínez de Osma, Comentario a la Ética de Aristóteles (1496). [Petri Osmensis In Libros Ethicorum Aristotelis Commentarii]. Introducción y selección de textos de Ana Cebeira, Serie de Pensamiento Español, Cuadernos de Anuario Filosófico, Servicio de Publicaciones de la Universidad de Navarra, Pamplona 2002.

9 Muñoz Delgado, V., Lógica, ciencia y humanismo en la renovación téológica de Vitoria y Cano, Instituto Francisco Suárez, CSIC, Madrid 1980. García y García, A., Muñoz Delgado, V, La «Summa» de Pedro de Osma sobre «La Politica» de Aristóteles, Instituto Francisco Suárez, CSIC, Madrid 1982.

10 González, G., Dialéctica escolástica y lógica humanistica de la Edad Media al Renacimiento, Ediciones Universidad de Salamanca, Salamanca 1987.

11 Rodríguez-San Pedro Bezares, L. E. (Coord.), Historia de la Universidad de Salamanca, I, Trayectoria y vinculaciones, Ediciones de la Universidad de Salamanca, Salamanca 2002. Están en curso de publicación, ya muy avanzados en la imprenta, los volúmenes, II y III.

12 Orrego Sánchez, S., La actualidad del ser en la «primera Escuela de Salamanca». Con lecciones inéditas de Vitoria, Soto y Cano. Eunsa, Ediciones de la Universidad de Navarra, Pamplona 2004.

13 Santiago-Otero, H. y Reinhardt, K., Pedro Martinez de Osma y el método teológico: edición de varios escritos inéditos, 95-100 y 103-114 respectivamente. En esta publicación además de estos dos textos, se recogen otros tres más. Todos ellos están transcritos del manuscrito 35 de la biblioteca capitular de Oviedo. Los datos que nos ofrecen del manuscritos en la página 37, entre otros, son los siguiente. El citado códice latino de 130 folios en papel, que miden $218 \times 132 \mathrm{~mm}$., están escritos en dos columnas en semicursiva gótica bien cuidada del siglo XV, con iniciales en rojo y en azul. Las tapas son de madera de $235 \times 155 \mathrm{~mm}$. El tejuelo con el título se ha perdido. En el primer folio y con la misma escritura gótica que se encuentra en el texto, se lee el título: Sermones magistro Oxomenxis. Más abajo, con escritura de mano posterior, se lee: Sermones y opúsculos del maestro Osma. El códice contiene 26 escritos de Pedro de Osma. Este manuscrito fue descubierto por Stegmüller, F., «Pedro de Osma. Ein Beitrag zur spanischen Universitäts-Konzils-und Kertzergeschichte», Römische Quartalscrift, 43 (1935) 205-66, y a ello aluden H. Santiago-Otero y K. Reinhardt, al igual que José Labajos Alonso.

14. $C E$, «Introducción», 22-23. $C M$, «Introducción», 43.

15 Comentario a la Ética de Aristóteles (1496). [Petri Osmensis In Libros Ethicorum Aristotelis Commentariij, 44-45. 


\section{PEDRO MARTÍNEZ DE OSMA (1424-80): OBRA Y SIGNIFICADO}

\section{a) Vida y obra:}

La Universidád de Salamanca por su posición de universidad eclesiástica al servicio de la cristiandad y de la Iglesia no era, ni podía ser ajena a los intentos de vertebrar la renovación que se estaba pretendiendo para la propia cristiandad en el siglo XV. La Universidad de Salamanca parece que se va dejando guiar por esta idea, pensando que era posible servir a ese proyecto de renovación y de ordenación de la unidad cristiana, y a la par de unidad nacional también y en esa dirección irían los esfuerzos de los grandes maestros salmantinos, que se aglutinan en torno al Concilio de Basilea: Alonso de Cartagena (1384-1456), Juan de Segovia (1393/95-1458), Alfonso de Madrigal, el Tostado o el Abulense (1401-1455).

Y tras los concilios de Ferrara-Florencia (1438-39) y de Basilea (1431-49), alertando sobre el nominalismo, además del platonismo como una nueva filosofia de la concordia para la cristiandad y que se iria manifestando en Nicolás de Cusa, pero sobre todo a través de la Academia Platónica de Florencia, en las universidades los empeños se iban a dirigir a encontrar una doctrina segura que pudiera vertebrar de modo seguro la filosofía y la teología. Platón o Aristóteles, esa era la tensión y el dilema. ¿Cómo repensar y responder mejor a las urgencias de la modernitas? ¿Cómo propiciar un mejor renasci? La apuesta en la Universidad de Salamanca se iba a decantar principalmente por Aristóteles y por Santo Tomás, aunque también se darían otras modulaciones. Es en esta coyuntura donde surge la obra de Pedro Martínez de Osma, integrando el humanismo y la herencia de Alonso de Cartagena, en pugna y alerta contra las novedades del nominalismo, y tratando de proponer una doctrina segura.

Pedro Martínez de Osma era de la diócesis de Osma, sin poder precisar si nació en Osma o en alguno de los pueblos de su arciprestazgo o del de San Esteban de Gormaz ${ }^{16}$. Se formaría y ejercería su actividad docente en la Universidad de Salamanca. Ingresa en el Colegio Mayor de San Bartolomé el primero de Mayo de 1444 y siguió los cursos en la Universidad, donde oyó, entre otros, al Tostado. En 1457 obtiene el grado de maestro en Artes a la par que desempeñaba la cátedra de Filosofia Moral (1457-63), que poco antes había ganado. En 1463 accede al grado de maestro en Teología y a la cátedra de prima de Teología, sucediendo a los dominicos que le habían precedido, Lope de Barrientos (1416- $i 36 ?)$ y Alvaro de Osorio (¿1436?-63). Pedro de Osma permanecería en dicha cátedra hasta el 30 de abril 1479, fecha en la cual salía hacia Alcalá de Henares ante el proceso que se le había abierto y comparecer ante la Junta de Teólogos el 15 de mayo. El dominico Diego de Deza le sucedería en dicha cátedra (1480-86), tras haber estado desempeñando diversas sustituciones.

Algunas de sus doctrinas teológicas acerca de la confesión y de las indulgencias no estaban exentas de polémica, y serían condenadas en dicha Junta de Teólogos de Alcalá en esa primavera-verano de 1479. La condena le apartó de la Universidad y de la ciudad de Salamanca, haciendo que se le manifestaran todo tipo de dolencias. Moría el 16 de Abril de 1480, confinado por la condena cerca de Salamanca, en Alba de Tormes.

Respecto a la filosofía, sus obras más destacadas son las siguientes: Compendium super sex libris Methaphisicae Aristotelis, escrito entre 1457-63 ${ }^{17}$; In Ethicorum Aristotelis libros commentarii, escrito entre $1457-60^{18}$; Summa super libris politicorum Aristotelis ${ }^{19}$; Responsio

16 Para Pedro Martínez de Osma remito a los estudios anteriormente señalados, principalmente a las «Introducciones» de José Labajos Alonso, $C M, 15-86$ y $C E$, 7-37.

17 Es el publicado en edición crítica latina por José Labajos Alonso, CM.

18 Sería corregido y editado por un discípulo suyo, el maestro Fernando de Roa, en Salamanca 1496. Ahora ha sido reconstituido con manuscritos en edición crítica latina por José Labajos Alonso, $C E$. 
ad quedam deliramenta duorum hujus temporis verbosistarum ${ }^{20}$; Dialogus in quo ostenditur fundamenta humanae philosophie quibus fulciuntur verbosiste in theologia deficere ${ }^{21}$; también cabe destacar su Tratado de Música.

\section{b) Humanismo y renovación}

Desde las anteriores preocupaciones, y en conexión con los grupos innovadores de la época, Pedro de Osma se iba a situar en la onda del movimiento humanista. Lo hacía en línea con la apuesta por la renovación que la propia Universidad de Salamanca había emprendido desde los Estatutos de Gramática de 1439, y en sintonía y tratando de emular a la Universidad de París, a la que surgía de la Reformatio Universitatis Parisiensis de 1452. Esta era la Salamanca que se renovaba y que se iría constituyendo en torno a Pedro de Osma y Antonio de Nebrija ${ }^{22}$.

Entre los rasgos más destacados de este impulso renovador que están presente en Pedro de Osma hay que destacar los siguientes ${ }^{23}$ :

\section{Su preocupación por la Gramática}

Pedro de Osma iba a apostar decididamente por la Gramática. Mérito indiscutible del humanismo era tanto la depuración de la forma literaria, el cuidado del estilo y del buen gusto, así como la preocupación por la Gramática. Y esta preocupación general se manifiesta en todo el Comentario a la Ética de Aristóteles, y además, y de modo concreto, en los dos problemas gramaticales, que inserta tras el comentario al «Prólogo» de Leonardo Bruni en el Comentario a la Ética ${ }^{24}$; en la referencia que en dicha obra hará a dos de los gramáticos más eminentes e influyentes, como Donato y su Ars Grammatica ${ }^{25}$ y Prisciano con su Institutio de arte grammatica ${ }^{26}$; en el encargo por parte del Cabildo salmantino de que realizara la oportuna corrección de un antiguo códice de la Biblia; y por haber sido un aval para Antonio de Nebrija en la consecución de la cátedra de Gramática en Salamanca en $1476^{27}$.

19 Según hace constar José Labajos Alonso, $C M$, «Introducción», 42, nota 116, en la Biblioteca Capitular de Pamplona se contiene una buena parte de la obra de Pedro de Osma. Los manuscritos 2, 23, 24 y 52 recogen algunas de las enseñanzas filosófico-naturales, éticas y teológicas que Pedro de Osma impartiera en la Universidad de Salamanca.

20 Cf. edición crítica señalada en, Santiago-Otero, H. y Reinhardt, K., Pedro Martínez de Osma y el método teológico: edición de varios escritos inéditos, CSIC, Madrid 1987, 95-100.

21 Ibidem, 103-114.

22 Fuertes Herreros, J. L., Estatutos de la Universidad de Salamanca. 1529. Mandato de Fernán Pérez de Oliva, Rector, Ediciones de la Universidad de Salamanca, Salamanca 1984, 34-37. Codoñer, C, González Iglesias, J. A. (Coord.), Antonio de Nebrija: Edad Media y Renacimiento, Ediciones Universidad de Salamanca, Salamanca 1994. García de la Concha, V., (Coord.), Nebrija y la introducción del Renacimiento en España, Ediciones Universidad de Salamanca, Salamanca 1983.

23 Cf., $C E$, «Introducción», 19-24, que sigo en algunos aspectos.

24 «An Grammatica fuerit tota ex hominum usu, an in aliqua sit naturalis» $\mathrm{y}$ «Quare apud universas linguas magis usui et quorumdam judicio standum erit quam usui ac judicio aliorum», ambas preguntas y respuestas en $C E$, 73 y 78 respectivamente

$25 C E, 44$. Cf. Codoñer, C., «Las Introductiones latinae de Nebrija: tradición e innovación», en Garcia de la Concha, V, Nebrija y la introducción del Renacimiento en España, 105-122, donde se estudia esta presencia de Donato y Prisciano en la Salamanca de Nebrija.

26. $C E, 75$ ss.

$27 A U S$, «Libros de Claustros», 2, fol.73v, 9 de enero, $C E$, «Introducción», 20, nota 33. 


\section{Apuesta por la Retórica}

El empeño de los humanistas por sustituir la Dialéctica por la Retórica se hace presente Pedro de Osma, también, a lo largo del Comentario a la Ética. Ya desde el mismo comentario al «Prólogo» del Aretino hace patente esto, y muestra su conocimiento y aprecio por la retórica aristotélica y ciceroniana ${ }^{28}$. Proclama su vuelta al Aristóteles de la argumentación retórica, que es la que emplea, por sus múltiples ventajas, en la Física, y en la Ética ${ }^{29}$. Y desde esa perspectiva, había iniciado, inclusive también, su comentario a la «Carta dedicatoria» de Leonardo Bruni.

\section{Vuelta a Aristóteles}

He indicado apuesta por la retórica y con ella y desde ella, ahora, vuelta a Aristóteles. Reencuentro con el Aristóteles humanista, con el Aristóteles descubierto y propugnado por Leonardo Bruni (137/74-1444), con el seguido y puesto de moda en Salamanca por Alonso de Cartagena, tras los titubeos en torno a 1430. Este Aristóteles es el que estará presente en Pedro de Osma, utilizando la versión del Aretino de la Ética a Nicómaco (1418). Era la vuelta a un autor antiguo, haciéndolo seguro desde una versión que había sabido alcanzar en la lengua latina una plenitud similar a la que tenía en las letras griegas, en línea con la apuesta por la renovación que la Universidad de Salamanca había hecho, tal como se ha indicado.

Pedro de Osma en su Comentario a la Ética de Aristóteles incorpora y comenta la «Carta dedicatoria» de Leonardo Bruni al Papa en la cual le dice que «traduje al latín con un cuidado especial los libros que tratan sobre las costumbres de Aristóteles, muy delicados y elegantes y necesarios para nuestra vida, pensando que nada podía hacer más útil que esto» ${ }^{30}$, alabando esta traducción de Bruni se preguntará, «¿qué puede haber más útil en la vida que conocer aquello por lo que dejamos de vivir para la muerte?, ¿qué por lo que distingamos nuestros caminos y actos?»31. Y lo mismo hará con el Prólogo del Aretino.

En éste, el propio Leonardo Bruni señalará los motivos que le impulsaron a traducir la Ética: «Hace poco decidí traducir al latín los libros de los Éticos de Aristóteles, no porque no hubiesen sido traducidos antes, sino porque habían sido traducidos de tal manera que parecian convertidos en bárbaros más que latinos. Pues consta que el autor de aquella traducción (cualquiera que al fin fuera éste, lo que es claro es que fue de la orden de los predicadores) sin duda no conocía suficientemente ni las letras griegas ni las latinas, pues en muchos pasajes entendió mal el texto griego y lo tradujo al latín tan pueril e ignorantemente, que hay que avergonzarse profundamente de tan supina y crasa rudeza; más aún ignorante con frecuencia de las palabras que tiene la latinidad óptima y muy probada y mendigando en nuestra opulencia no sabe traducir al latín la palabra griega, como desesperando y carente de plan de tal manera lo despacha que las deja en griego, así resulta semigriego y semilatino, estando falto en una y otra lengua y no completo en ninguna de las dos. Qué decir de la transformación de la oración, nada hay más desordenado, nada más pervertido ${ }^{32}$.

El ejemplo y estímulo para haber emprendido su traducción le venía a Leonardo Bruni al ver lo que supieron hacer Plauto, Virgilio y Cicerón, «que Plauto y Terencio, poetas cómicos, que tratan de asuntos muy ligeros, han vertido las fábulas de Menandro tomadas del griego de tal manera que expresan todo incluso lo muy minucioso, pero no se sirven de palabras griegas

28 CE, 52ss.

$29 C E, 24$ y 96 ss

$30 C E$, «Carta de Leonardo Bruni al Papa», 43.

31 CE., «Comentario a la Carta de Leonardo Bruni al Papa», 46. La traducción de las citas del «Prólogo y comentario de Leonardo Bruni»), han estado al cuidado de Esteban Monjas Ayuso.

$32 C E$, (Prólogo de Leonardo Bruni», 47. 
ni les falta una elegancia extraordinaria ni un adorno. Cicerón también en estos libros en los que puso por escrito estos tratados de Aristóteles, nunca echó en falta la facilidad de expresarse, nunca la riqueza del discurso» ${ }^{33}$.

$Y$ en el comentario de Pedro de Osma a este «Prólogo» se manifestaba conforme con Bruni y aceptaba desde dicha traducción, ya lavada de las manchas que pesaban sobre Aristóteles, iniciar gozoso su comentario a la como nueva Ética, ya que «ahora tenemos estos libros traducidos por Leonardo al latín» ${ }^{34}$.

Con lo cual tras un «Prólogo» propio como «Introducción» a la Ética, tras situarla en el lugar que le corresponde dentro de la ordenación de las ciencias, dentro de la filosofía práctica civil ${ }^{35}$, iniciará su comentario a la Ética a Nicómaco, siguiendo cada uno de los diez libros de Aristóteles según la traducción de Leonardo Bruni ${ }^{36}$, y sirviéndose de la gramática y de la retórica para este comentario, señalando que éstas pertenecen al ámbito de la filosofia práctica civil ya que «eleganter enim et ornate dicere, quorumprimum est grammaticae, secundum rethoricae, ad hominem mores non modicum attinet $\rangle^{37}$, y dejando fuera a la dialéctica, aduciendo que no cae dentro de este ámbito de la fillosofía práctica, sino de la filosofía especulativa ${ }^{38}$. Ahora bien, ¿qué papel le queda o le asigna a la dialéctica desde esta ordenación?, ¿qué consideración le merece la dialéctica a Pedro Martínez de Osma? Dejo, aquí, planteadas estas preguntas a las que iré dando respuesta.

\section{Rechazo de la dialéctica y del nominalismo}

Quizá ya hemos puesto el posible fundamento para determinar la consideración que desde una ordenación de los saberes, la dialéctica, le merecía a Pedro de Osma. Ahora bien, ¿cuál es la razón qué le lleva a considerar de este modo a la dialéctica, esto es, cayendo dentro de la filosofía especulativa, pero a la par dejándola sin contenido? La respuesta está arrastrando inevitablemente la complejidad y las tensiones de su propio momento histórico. Esto ayudar a comprender la Respuesta a algunos disparates de dos verbosistas de esta época, y el uso del género irónico que utilizará.

Pedro de Osma estaba en una Universidad, la de Salamanca, que tras el Concilio de Basilea estaba apostado por una renovación de los saberes, y por dar respuesta adecuada a los problemas de su tiempo desde doctrinas que fueran seguras. Las consecuencias a que había ido llevando el nominalismo habían aflorado en Basilea. Se había percibido que desde el conciliarismo se había tensionado y prolongado la solución al Cisma de Avignon, provocando dolorosas situaciones para la Iglesia.

Ahora se estaba en una época nueva, se estaban ensayando, también, otras filosofias volviendo a Platón, se pretendía restaurar la cristiandad, articularla en el espacio de la todavía vieja imago mundi.

$\mathrm{Y}$ era la hora de las filosofias seguras, de los autores antiqui frente a los moderni, de los reales frente a nominales o terministas, de los doctores antiqui frente a los doctores renovados (renovatorum). Iba a ser la pugna entre Platón, y entre Aristóteles; el Comentador Averroes, Alberto Magno, Santo Tomás de Aquino, Egidio de Roma, Alejandro de Ales, Scoto y San Buenaventura, frente a Guillermo de Ockham, Juan de Mirecourt, Gregorio de Rímini, Juan Buridano, Pedro de Ailly, Marsilio Inghen, Adam Wodeham, Juan Dorp y Alberto de Sajonia.

\footnotetext{
$33 C E$, «Comentario al Prólogo de Leonardo Bruni», 56

$34 C E$, «Comentario al Prólogo de Leonardo Bruni», 73.

$35 C E, 81-85$.

$36 \quad C E, 89-654$.

$37 C E, 85$.

$38 C E, 85$ : «Mihi quidem videtur quod Dialectica sub philosophia speculativa continetur. Rethorica vero et Grammatica sub civili».
} 
Y era la lucha entre la dialéctica escolástica nominalista y la lógica humanista, entre nominalismo y humanismo, o entre renovación nominalista o humanista. $Y$ esto era el debate y las tensiones que se vivían en la Salamanca de Pedro de Osma ${ }^{39}$.

\section{También Lorenzo Valla y Antonio de Nebrija}

Como señalaba Leonardo Bruni, «me pregunto cuál habrá sido la causa de alejarse de la práctica de hablar de Séneca, Cicerón, Boecio, Lactancio, Jerónimo y de otros de los nuestros ${ }^{40}, \mathrm{y}$ también del mismo Aristóteles, pues como comenta Pedro de Osma, «trata pues el Aretino de mostrar que Aristóteles había sido sabio no sólo en sabiduría sino también en elocuencia.Y esto lo prueba doblemente: primero por la autoridad de Cicerón que en el asunto del que tratamos no debe ser tenido en poca consideración. Dice, pues, en primer lugar, atqui, esto es, ciertamente Cicero ipse testatur in multis locis de sus libros, es decir, en la primera Retórica, en las Cuestiones Tusculanas y en el Libro sobre el Orador, testatur, digo, Aristotelem fuisse studiosum eloquentiae. Y dice Cicerón que Aristóteles había combinado el arte de la sabiduría con el arte de la elocuencia, esto es, con la retórica. Pues es aquella que nos enseña a hablar pertinentemente. Et libri eius aquí, en segundo lugar, prueba lo mismo sobre el estilo de Aristóteles, diciendo et libri eius, evidentemente, de Aristóteles scripti en graeco cum summo studio eloquentiae, esto es, de elegancia y ornato (eloqui es hablar con elegancia y belleza), elocuencia elegante y locución adornada. Declarant, esto es, muestran, luculentissime, esto es, clarísimamente, esto, a saber, que Aristóteles ha sido no solo sabio en sabiduría, sino también en el arte de decir, que los griegos llaman retórica pero los nuestros llaman elocuencia. Así pues arguye el Aretino del efecto a la causas ${ }^{4 !}$.

Y es la argumentación retórica la que se debe preferir, es la que en sintonía con Cicerón en la Rhetorica ad Herennium, está empleando Leonardo Bruni, «estas, pues, son las partes de una muy perfecta argumentación retórica y el Aretino se había servido al presente de esta perfectísima argumentación $\rangle^{42}$.

Era, también, la nueva orientación que Lorenzo Valla $(1407-1457)^{43}$ había introducido en las Elegantiarum linguae latinae libri sex, que comienza a circular desde 1440 (Roma 1444; Venecia 1471) y en las Disputationes dialecticae que por estos años compone, 1439-49 (1499). Había de alguna manera que refundar la filosofía sobre la base del uso del latín clásico y desterrar por innecesaria la lógica escolástica, que era un arte de guerrear, tal como se cultivaba en las escuelas, haciendo que pasara a primer plano la retórica frente a la dialéctica. Será la lógica o dialéctica una parte de la retórica. El razonamiento escolástico, que tendía a la verdad y la certeza es sustituido por la argumentación retórica, que se dirige a la persuasión y hacia la mayor probabilidad. Por ello, desde ese uso de la lengua de los grandes autores latinos y del uso ordinario de la lengua de los hombres había que iniciar esa nueva época que tocaba edifi-

39 Muñoz Delgado, V., Muñoz Delgado, V., Lógica, ciencia y humanismo en la renovación teológica de Vitoria y Cano, ya referenciado. Id., «Ciencia y filosofia de la naturaleza en la Península Ibérica (1450-1600)», Repertorio de Historia de las Ciencias Eclesiásticas en España, vol. 7, Salamanca 1979, 67-149. Id., "Nominalismo, lógica y humanismo", El erasmismo en España. Estudios de literatura y pensamiento hispánicos, Sociedad Menéndez Pelayo, Santander 1986, 109-174. González, G., Dialéctica escolástica y lógica humanística de la Edad Media al Renacimiento, ya referenciada.

$40 C E$, «Prólogo de Leonardo Bruni», 49.

$41 C E$, «Comentario al Prólogo de Leonardo Bruni», 54-55.

$42 C E, 53 . \mathrm{Y}$ estas eran las partes que Cicerón había señalado, un poco antes, en esta misma página: «Secundum Tullium in Rethorica ad Herennium, partes sunt quinque, scilicet, propositio, ratio, rationis confirmatio, exornatio, conclusio..»

43 Fernández López, J., Retórica, humanismo y filología: Quintiliano y Lorenzo Valla, IER (Instituto de Estuios Riojanos), Logroño 1999. Valla, L., De linguae latinae elegantia, 2 vols. Introducción, edición, traducción y notas por Santiago López Moreda, Universidad de Extremadura, Cáceres 1999. 
car tanto filosófica como filológicamente. Como decía Valla: «Debido al amor que profeso a mi patria y a todos los hombres, y por la trascendencia del problema, conviene exhortar a todos los que cultivan la elocuencia y gritarles como desde la cima de un monte y hacer resonar, como dicen, el toque de alerta. ¿Hasta cuándo... consentiréis que la latinidad esté oprimida por la barbarie? ¿Hasta cuando contemplaréis con ojos duros y casi despiadados que todo esté profanado?, ¿acaso hasta que apenas queden restos de los cimientos? $\rangle^{44}$.

Ese sería también el espíritu que Antonio de Nebrija al regresar a España en 1470, y tras estar algún tiempo en Sevilla, traería al ser llamado en 1475 a la Universidad de Salamanca, después de haber estado entre 1460-1470 en el Colegio de los Españoles de Bolonia, donde se empapa del humanismo que allí se respiraba y conoce las Elegantiae de Valla. Y ese modelo es el que se filtraría en Salamanca, también, en sus Introductiones latinae explicatae, 1481 y en su Differentiae excerptae ex L. Valla, Nonio Marcello et Servio Honorato, ca. 1498. Ese era el Antonio de Nebrija que acude a Pedro Martínez de Osma para que lo avale como testigo en la oposición a la cátedra de Gramática, y el Pedro de Osma que no duda en hacerlo y alabarlo, tal como se ha indicado, y esa la Universidad de Salamanca de esos años en la cual Nebrija toma posesión como catedrático de Gramática.

\section{NUESTROS TEXTOS A TRADUCIR}

En esta situación, ya muy concreta y ceñida, heredera de Basilea, con los aires de esta renovación humanista, en pugna con la dialéctica y el nominalismo o con la renovación nominalista, y ofreciendo un nuevo método, es donde afloran los escritos de Pedro de Osma, que expresaban, también, la inquietud de la cristiandad, Respuesta a algunos disparates de dos verbosistas de esta época, y Diálogo en el que se muestra que los fundamentos de la filosofia humana, en los que se apoyan los verbosistas, generalmente fallan en la teologia ${ }^{45}$, y que traducidos se ofrecen.

El nominalismo como movimiento intelectual se había irradiado por todas las Universidades, desde París, pasando por Oxford, Viena..., y había llegado, también, a Salamanca. E igualmente desde el humanismo ${ }^{46}$ y desde las Universidades se le trataba, ahora, de combatir, o mejor neutralizar, promoviendo el descubrimiento y el çltivo de la verdadera Sabiduria desde una doctrina y un método seguros.

La última condena contra el nominalismo se había producido en la Universidad de París por el Decreto Real de Luis XI, del 1 de marzo de 1474, por el cual se prohibía rigurosamente el nominalismo. En dicho decreto se señalaban las líneas peligrosas que el nominalismo había emprendido y en las cuales habían caído algunos maestros: cultivaban doctrinas inútiles, superfluas, estériles y peligrosas, iban tras novedades, se habían apartado de las doctrinas sólidas y saludables de los Padres y de los doctores reales, y propiciaban una vida disoluta y costumbres corrompidas entre los estudiantes. Y frente a los doctores renovados se proponían los doctores reales, que señalamos anteriormente.

44 Valla, L., De linguae latinae elegantia, I, 63.

45 Cf. referencia dada en notas 13 y 14.

46 Los personajes y obras hacia quienes principalmente se dirigen los ataques de estos humanistas son: Guillermo de Shyreswood (+1249), Introductiones in Dialecticam. Ricardo Swineshead (Suisseth, Suisset, el «Calculador») (Colegial de Merton 1348), Liber conclusionum. Guillermo Heytesbury (+ hacia 1380), Regulae solvendi sophismata. Radulfo Strodo (hacia 1370), Consequentiae et obligationes. Ricardo Ferabrich (hacia 1370), Consequentiae. Pedro Hispano (1210/20-1277), Summulae Logicales. Guillermo de Ockham (hacia 1285-1349), Expositio aurea super veterem artem, y Summa totius Logicae. Walter Burleigh (1275-después 1349), De puritate artis logicae. Juan de Buridán (hacia1295-1358), Summula de Dialectica. Alberto de Sajonia (hacia 1316-1390), Perutilis Logica. Pablo de Venecia (Paulus Venetus) (+ 1429), Logica magna, y Logica minor. 
La fecha de composición de estos manuscritos de Pedro de Osma, que carecen de fecha, habría que situarla en el ambiente de esta última prohibición, después de 1474, ya que parece hacer referencia a esta condena: «esta posición de los doctores sagrados jamás estuvo muerta, jamás enterrada, sino resplandeciente y viva. Vive hoy también más que nunca y está en vigor casi en todo el mundo en las universidades más prestigiosas, sobre todo en París, donde se rechaza a los que sostienen lo contrario, pues están excomulgados» ${ }^{47}$, y entre el 30 de abril de 1479 , fecha en la cual se ve forzado a salir de Salamanca camino de Alcalá para comparecer ante la Junta de Teólogos por el proceso que se le había incoado por sus doctrinas sobre la Confesión, habiendo dado sus últimas clases entre los días 24 a 30 de abril de dicho año ${ }^{48}$. De otra parte, el Decreto Real de Luis XI sería revocado en 1481.

El término general que empleará para dirigirse y criticar a estos nominalistas y a su metodología, será el de «verbosismo», caracterizado por la búsqueda de palabras nuevas, vacías, carentes de tradición, envueltas en sutilezas, formalidades, verbosidades, por no buscar la verdad sino por verbalizaciones artificiosas, y los denominará vociferadores, verbosistas, formalistas, nuevos doctores, modernos, cultivadores de palabras, perturbadores de toda doctrina, viles, fumosos o fumosistas ${ }^{49}$. Ahora bien, ¿qué papel le queda o le asigna a la dialéctica desde esta ordenación?, ¿qué consideración le merece la dialéctica a Pedro Martínez de Osma? No otro que un servicio obsequioso a la fe y al propio descubrimiento de la Verdad y Sabiduría, tal como aparecerá en nuestros textos.

\section{a) "Respuesta a algunos disparates de dos verbosistas de esta época»}

La Respuesta a algunos disparates de dos verbosistas de esta época, es una recreación satírica al estilo del Elogio de la locura, 1509 (1511), o de La nave de los necios (1494) de Sebastián Brant, o de las críticas que había efectuado Rodrigo Sánchez de Arévalo, unos años antes, en el Speculum vitae humanae, $1468^{50}$.

Imagina una suerte de combate admirable, tal como podía evocar la Psycomaquia de Aurelio Prudencio, cuyas obras unos años más tarde iba a publicar Nebrija ${ }^{51}$, o como, de otra manera, Francesco Colonna en su Poliphili Hypnerotomachia (1499) nos iba a dejar. Es un torneo, disputa o debate singular, en el cual se van a batir, con sus divinos nombres, los príncipes de los verbosistas de esta época. El espectáculo es grandioso, la acción se presenta trepidante y la ironía finísima e inteligente.

Los nombres de estos dos príncipes famosos, desde esta recreación lucianesca, con bastante probabilidad son los maestros Pedro Caloca, para «Licet loca» (Ca loca), esto es, lo que podía corresponder actualmente, y de modo despectivo, a wáteres o servicios, y Pedro de Ocaña, para «O caña» ( Oh caña, oh caña!).

47 Responsio ad quedam deliramenta duorum huius temporis verbosistarum, 97, § 57-61. Respuesta, 97, cito por la paginación introducida en negrita en la traducción efectuada en las págs. 311-314 de esta Revista.

48. CM, «Introducción», 39; en las páginas 37-39 se detallan las fechas de su actividad docente, siguiendo los Libros de Claustros de la Universidad de Salamanca.

49 Estas expresiones, además de en estos textos nuestros, Respuesta, sobre todo en 100 (Responsio, 100, $\$ 158$ 166), y Diálogo, alguna, la de verbosistas, la encontramos en una repetitio relacionada con el Diálogo, Repetitio de comparatione deitatis, propietatis et persone, cf. en Santiago-Otero, H. y Reinhardt, K., Pedro Martínez de Osma y el método teológico: edición de varios escritos inéditos, 47-91, entre otras, en $71,78,79,80,89,91$, y la de vociferadores en 69.

50 Fuertes Herreros, J. L., «Filosofia de la historia y utopía en el XV. Una aproximación al Speculum vitae humanae (1468) de Rodrigo Sánchez de Arévalo», en Pensamiento Medieval. Homenaje a Horacio Santiago-Otero, (Coord. José María Soto Rábanos), CSIC, Madrid 1998, vol. II, 1317-46.

51 Aurelli Prudentii Clementis viri consularis libelli cum commento Antonii Nebrissensi, Lucronii, per Arnaldum de Brocario, 1512. 
En efecto, el franciscano Pedro de Caloca había concurrido con Pedro de Osma a la oposición a la cátedra de Prima de Teología en 1463, en la que resultó vencedor Pedro de Osma, y fue su acusador en el proceso de Alcalá seguido contra Pedro de Osma. Y según Esperabé y Arteaga, Pedro de Caloca ocuparia la cátedra de Visperas de Teología entre $1454-i_{1487}$ ? ${ }^{52}$. Pedro de Ocaña, pertenecía al convento de los dominicos de San Esteban de Salamanca, y se distinguía por sus tendencias nominalista así como por su desprecio a Santo Tomás. Tuvo la disputa de unas Conclusiones sobre la esencia y paternidad de Dios con Pedro de Osma en dicho convento de los dominicos en la festividad de San Juan Bautista y aparece, también, como acusador de Pedro de Osma en el proceso de Alcalá ${ }^{53}$.

Ahí tenemos a los personajes, saltando a ese combate singular de destreza dialéctica y de ingenio. Primero, a «Licet loca», y en segundo lugar, a «O caña».

El príncipe «Licet loca» blandía sus centelleantes armas y «lugares»: tología por teología, tólogos por teólogos, puntos de vista enterrados, ultrajes, injurías, a mí mis doctores, razonamientos, silogismos, arguyo por los principios de la lógica, tercer modo de la primera figura, es decir, Darii, la consecuencia por la mayor, la menor está clara, palabras ultrajantes, verbosistas, vociferadores.

La respuesta del maestro Pedro de Osma, intentando sobreponerse a tanto espanto y aturdimiento producido por los encantamientos y por las nuevas estratagemas empleadas por «Licet loca», comenzará por poner orden. Orden en un mundo que aparecía como mundo al revés, que había ido tras las novedades que, ahora, en París, hasta estaban excomulgadas, y había olvidado la santidad y la verdad. Verdad y santidad que no se viste del artificio, ocultando el peligro del escorpión con que se representaba a la dialéctica, sino que se muestra en la propia sencillez de la verdad, la cual hasta puede aparecer como asinina, pero que de ninguna manera lo era. Más bien resultaba ser ciudadela inexpugnable, protegida por los escudos de la torre de David.

Hay que ir a la verdadera sabiduría, renunciando a creer que su poder está en los nombres, hay que dejar de ser vociferadores, portadores y cultivadores de nombres, y había que volver, derechamente, al canon sagrado, a los santos, a los doctores seguros, a Santo Tomás, doctor antiguo, lleno de luz, lúcido, claro y divino, había que volver, en definitiva, a la verdadera sabiduría, a beber de las fuentes del Espíritu Santo.

¿Podía haber en esto injuria o ultraje alguno? ¿Acaso era injuriar el advertir, el corregir, el alertar, el señalar los engaños de la imaginación, el llamar a la verdadera sabiduría?

Tras esto, en este combate singular apareció relumbrante el príncipe «O caña», blandiendo sus cañas que supo lanzar contra el maestro Osma. Lanzó la de insolente, la de presuntuoso, la de no ser verdadera su doctrina y la de no ser la propia de un sabio. Hasta le lanzó una especialmente envenenada, que decía: maestro jovenete e ignorante, no sabe nada, a él mismo se lo he oído decir.

El combate había llegado en este punto a su momento álgido. Se desvelaban los puntos de seguridad de cada contendiente. Formalistas contra las escuelas del santo doctor, doctores moderni contra doctores antiqui. Los que querían emitir juicios sobre el supremo Creador y los que partían de la debilidad y fragilidad de la razón humana para las cosas divinas. La de los que se engañaban con el humo de una cierta imaginación de la verdad y la de los que recurrían a la fe y a la tradición segura de la Iglesia. La de los perturbadores de toda la sana doctrina con sofismas y arte dialéctico, y la de la tradición asentada y perfeccionada en los santos doctores y comentadores.

52 Esperabé y Arteaga, E., Historia pragmática e interna de la Universidad de Salamanca, Salamanca 191417, II, 246. Santiago-Otero, H. y Reinhardt, K., Pedro Martínez de Osma, 95. CE, «Introducción», 23.

$53 C E$, «Introducción», 23. $C M$, «Introducción», 44 y nota 128, donde José Labajos transcribe las Conclusiones. 
¿Acaso no era sabio el que seguía el camino de la verdadera sabiduría? ¿El que reconocía los límites de la razón? ¿El que se atrevía a someter a crítica a la razón?

¿Se podía decir que se cometía injuria contra los que practicaban lo contrario? ¿Acaso no eran ciertamente: vociferadores, verbosistas, viles, fumosos o fumosistas o como más les gustara?

Así concluía este combate, y no ya de azar dudoso, ni de amor o desamor en sueño, sino trazando caminos seguros para ordenar el tiempo presente. Y Pedro de Osma lo cerraba, sellándolo con la respuesta que los verbosistas en su príncipe, el maestro Ocaña, merecía: ¡Oh caña!, joh caña!, joh caña!

¿Y cómo resonaban los ecos de la Escritura! «¿Qué salisteis a ver al desierto? ¿Acaso una caña agitada por el viento o a un profeta?... Yo os digo que más que a un profeta. Éste es de quien está escrito: 'He aquí que yo envío a mi mensajero' ${ }^{54}$. ¿Qué decir de los verbosistas y de su príncipe el maestro Ocaña? Solamente la respuesta que merecen: ¡Oh caña, oh caña, oh caña!

¡Enderezad los caminos, volved al camino recto! ¡Id tras el profeta, en pos del santo doctor, de Santo Tomás, tras el mensajero que os fue dado para renacer en estos nuevos tiempos!

\section{b) «Diálogo en el que se muestra que los fundamentos de la filosofia humana, en los que se apoyan los verbosistas, generalmente fallan en la teología)}

El segundo de los textos que se presenta, es el Diálogo en el que se muestra que los fundamentos de la filosofia humana, en los que se apoyan los verbosistas, generalmente fallan en la teología.

$\mathrm{Y}$ es un diálogo que pertenece a dicho género, muy estimado en el Renacimiento ${ }^{55}$. Era cercano en el tiempo al Speculum vitae humanae de Rodrigo Sánchez de Arévalo, que también está escrito a manera de dialógo ${ }^{56}$.

Pedro de Osma nos va proponer este texto como un diálogo didáctico ${ }^{57}$ entre en un maestro y un discípulo: «Y como esta cuestión se desarrolla entre maestro y discípulo, vamos a presentarla en forma de diálogo entre los dos: el discípulo preguntando y arguyendo, el maestro a su vez, respondiendo y como intérprete de la verdad. En primer lugar, como exige el orden correcto, empezará el discípulo arguyendo, luego, subsigue el maestro respondiendo ${ }^{58}$.

Pero es un diálogo, que dividido en cuatro partes, parece poner al día o encubrir la técnica y estructura del artículo de la Suma de Santo Tomás, adaptándola a los nuevos tiempos. Así contiene, en primer lugar, el planteamiento de la cuestión, siendo determinada de modo preciso por el maestro. Luego, en segundo lugar, se exponen los argumentos en contra, esto es, por el discípulo a través de los «Argumentos del discípulo» ${ }^{59}$, con lo cual nos hemos introducido de lleno en el diálogo. Así se llega, en tercer lugar, a la determinación o solución de la cuestión planteada, tal como lo hace el maestro en el propio Diálogo, «Cuerpo de la cuestión» ${ }^{60}$, $\mathrm{y}$, por último, la cuarta parte o solución de los argumentos propuestos, que será nuevamente a cargo del maestro, «Respuesta del maestro a cada argumento del discípulo» ${ }^{6 !}$.

54 Mt., 11, 7-10.

55. Gómez J., El diálogo en el Renacimiento español, Cátedra, Madrid 1988.

56 Fuertes Herreros, J. L., «Filosofia de la historia y utopía en el XV. Una aproximación al Speculum...», 1322-23.

57 Gómez, J.: «En el diálogo didáctico, considerado como género literario, los interlocutores, el tiempo y el espacio están al servicio de las ideas que, a su vez, dependen del proceso discursivo, lógico o retórico de la argumentación», 13.

58 Diálogo, 103. Cito por la paginación introducida en negrita en la traducción de este texto de Pedro Martínez de Osma, efectuada más adelante en este mismo número de la Revista.

59 Diálogo, 105.

60 Ibidem, 107, final.

61 Ibidem, 109. 
El Diálogo comenzará, tal como la recreación lo exigía, por tratar de determinar la cuestión, y que venía en forma de pregunta tras una supuesta exposición o disputa, «si en las personas divinas se puede encontrar alguna diferencia que sea mayor que la diferencia según el nombre o la razón y menor que la diferencia esencial $\rangle^{62}$, que evocaba lo ya tratado en la $R e-$ petitio Magistri de Oxma de comparatione deitatis, propietatis et persone, y a la que está aludiendo en este Diálogo.

Así parecía que se quería plantear y clarificar este dificilísimo tema. Pero, en definitiva, venía tocado de una pregunta previa, que era también la que había dejado resuelta en la Respuesta con el rechazo del nominalismo, esto es, «preguntaban algunos de los discípulos si, por la fuerza de alguna explicación natural o por la filosofía humana» era posible responder a esa pregunta. Por eso, ante la propia imposibilidad ya supuesta, mejor era plantearla de un modo desde el cual se obligara nuevamente a la razón a caer rendida ante la fe. «Ahora bien, estas tres cuestiones de diferentes discípulos pueden resumirse en una sola: si por la fe, o por la filosofia humana, o por ambas a la vez, la inteligencia del viador, que no está especialmente iluminada para esto, podría demostrar en las personas divinas alguna diferencia mayor que la diferencia según el nombre o la razón o menor que la diferencia esencial $»^{63}$.

Era un modo de someter a prueba a razón, era una crítica de la razón especulativa, o de las pretensiones de la razón nominal, de los verbosistas, y a la vez mostrar cómo era sobrepasada, dentro de este ámbito espiritual cristiano en el cual nos estamos moviendo.

Pero desde la ficción del diálogo, había que determinar la cuestión, para fijar los argumentos a favor o en contra, o mejor, para poderse dar la palabra.

La postura del maestro, la tesis que a través del diálogo irá exponiendo Pedro de Osma, será bien clara y así se la hará saber desde el principio al discípulo, «no hay nada con lo que la inteligencia del viador, que no está especialmente iluminada para esto, pueda demostrar en las personas divinas alguna diferencia que sea mayor que la mera diferencia según el nombre o la razón, pero menor que la esencial» ${ }^{64}$.

Con lo cual se pasaba a la segunda parte del Diálogo. Los hasta quince argumentos del discípulo encaminados a aportar la posibilidad contraria y a escudriñar este tema, a pesar de sus esfuerzos, más acabarán produciéndole desazón que tranquilidad, o mejor, era toparse ante los límites de la razón y descubrir su impotencia al verse sobrepasada. «¿Hay más cosas que te preocupen?», le preguntará el maestro tras el último argumento, el número quince.

Así se posibilitaba el entrar en el «Cuerpo de la cuestión, esto es, en la tercera parte, y la intervención del maestro, que con mayor claridad volverá a señalar que los fundamentos de la filosofia humana y los argumentos de la razón fallan de modo general en teología y de modo muy ostensible en este caso concreto. «Pues bien, para que puedas ver más fácil y claramente que todas las cosas dichas antes no son demostraciones, sino unos sofismas, que te engañan a ti y a los demás, hay que hacer dos consideraciones. En primer lugar, que las reglas de los filósofos, esto es, las reglas de la razón humana fallan a propósito de las personas divinas en gran parte, lo que vamos a manifestar en algunas de aquéllas como ejemplo, de manera que será fácil extrapolarlo a las demás» ${ }^{65}$.

Llegados a este punto del Diálogo el discípulo va a requerir del maestro que le resuelva sus argumentos, o mejor, dada ya por desechada la vía de la filosofia por sus sofismas y contradicción permanente, que se los muestre desde la mejor y plena compresión de la fe. Y es lo que hará en esta cuarta parte, «Respuesta del maestro a cada argumento del discípulo» ${ }^{66}$, res-

62 Ibidem.

63 Ibidem.

64 Ibidem.

65 Ibidem, 107.

66 Ibidem, 109. 
pondiendo a cada uno de los quince argumentos expuestos en la segunda parte. Y el maestro lo hará desde el modo que lo puede hacer, esto es, partiendo de la fe y desde ese servicio obsequioso que la razón ya está dispuesta a ofrecer a la fe y a revelación. Por eso, ahora, su exposición acudirá a beber de las frescas aguas de las fuentes seguras de la Revelación, de la Escritura Sagrada, de los santos Padres, de la tradición y de la doctrina de la Iglesia.

Pedro de Osma, para dejar más en claro cuál era el propósito último de este Diálogo o de la renovación que pretendía, sabrá introducir a través del propio diálogo entre el maestro y el discípulo en sus últimos párrafos, tal como los he numerado, y conteniendo esta advertencia: «Guarda esto en tu mente contra los verbosistas, que estando no lejos del error ponen escándalo en todos los lugares con sofismas y el arte dialéctico ${ }^{67}$.

Así, tras haber mostrado las insuficiencias de la razón, la necesidad de ir tras la verdadera sabiduría ${ }^{68}$, los ámbitos y fundamentos distintos en los que se mueve la fe y la razón, el distinto método que le es propio a cada una, concluía este Diálogo con esa propuesta de renovación humanista, que abandonando la vanidad de la dialéctica y las imaginaciones de la razón, tal como proponían los nominalistas, fuera capaz de construir la nueva cristiandad, que se estaba soñando desde:Basilea ${ }^{69}$.

\section{CONCLUSIÓN}

$Y$ así hemos llegado al final de esta presentación que ha tenido como propósito presentar unos textos, o más bien ha sido la excusa para disfrutar de unos textos, que saben expresar las tensiones de una época en búsqueda de la que soñaban fuera la mejor ordenación para la cristiandad.

En esta búsqueda y reflexión sobre su modernidad desde las experiencias dolientes de este largo siglo, Pedro de Osma desde su Universidad, la de Salamanca, había sabido sintonizar con el humanismo e ir desde aquí, hasta en diversos géneros literarios, a las fuentes, a los antiqui. Así, señalando y abriendo el camino, con método seguro y alertando sobre los peligros de la dialéctica y del nominalismo, presentaba una filosofia y una teología renovada.

La suerte estaba echada. Hasta Francisco de Vitoria quedaba todavía un largo recorrido. A lo lejos, la Escuela de Salamanca y a Vitoria abriendo la Suma de Santo Tomás para comentarla, y aquí, en estos textos, a Pedro de Osma oteando el tiempo de la historia desde su presente, desde un humanismo que pretendía ir no «Ad Narragoniam» en La nave de los necios de Sebastián Brant, sino alcanzar la verdadera Sabiduría.

José Luis Fuertes Herreros

Facultad de Filosofía

Universidad de Salamanca Campus «Miguel de Unamuno» 37007 Salamanca

67 Diálogo, 113, [1].

68 Diálogo, 113, [1]: «Y a esto parece que ofrece una prueba no pequeña la gran cantidad de herejes, porque, aunque en la sapiencia, o, mejor, en la insipiencia humana parecen muy agudos, sin embargo, no las vieron ni en sueños. Dios, en efecto, ha revelado a los pequeños, lo que ha ocultado a los sabios, como se dice en Mateo 11 y en Lucas 10 ».

69 Ibidem, 114, [6]: «Así pues, la inteligencia humana, considerando sólo las cosas creadas, no podrá ni ver ni sospechar esta distinción de las personas divinas en unidad esencial. Así pues, en estas cuestiones y otras semejantes no hay que recurrir de ninguna manera a la vanidad de la dialéctica, ni a las imaginacionés de la razón humana, como hacen los verbosistas, sino a la santa madre Iglesia, para cuya utilidad y honor he dicho esto. Demos gracias a Dios». 